\title{
Reference data for bone speed of sound measurement by quantitative ultrasound in healthy children
}

\author{
Anjumanara Omar $\cdot$ Serap Turan $•$ Abdullah Bereket
}

Received: 15 February 2006 / Accepted: 20 July 2006 / Published online: 28 September 2006

(C) International Osteoporosis Foundation and National Osteoporosis Foundation 2006

\begin{abstract}
Introduction The aim of the study was to establish reference data for tibia and radius bone ultrasonic speed of sound (SOS) measurement in our pediatric population, and to compare the results with original (built-in) reference data. We also investigated the relationship between SOS Z-scores and anthropometric parameters and bone markers.

Method SOS was measured in 463 healthy children (116 years) for the tibia and radius using Omnisense 7000 device (Sunlight Medical Inc., Israel). Height, weight, calcium intake, serum alkaline phosphatase and osteocalcin concentrations were obtained.

Results Age- and gender-specific mean speed of sound (SOS) values that describe SOS changes at the tibia and radius were demonstrated. Reference data obtained in our population was very similar to the built-in reference data obtained in Israeli children except for the slightly lower radial SOS values in our population after 8 years of age. A triphasic pattern was observed with a steep increase in SOS values at $0-6$ year and at puberty, with a steady period during childhood for both genders at both sites. SOS Z-scores obtained in both measurement sites showed good agreement (girls $r=0.40, p<0.0001$; boys $r=0.33, p<0.0001$ ). No correlation was detected between SOS Z-score and height
\end{abstract}

\footnotetext{
A. Omar $\cdot$ S. Turan $\cdot$ A. Bereket

Department of Pediatric Endocrinology,

Marmara University School of Medicine,

Tophanelioglu Caddesi 13/15,

Altunizade-Istanbul 81190, Turkey

A. Bereket $(\square)$

Bozkir Sokak 4/7,

Selamiçeșme-İstanbul 81030, Turkey

e-mail: abereket@e-kolay.net
}

SDS, calcium intake or osteocalcin levels. SOS Z-score correlated negatively with weight SDS in girls and serum alkaline phosphatase in boys.

Conclusion We report the reference data for SOS in healthy Turkish children, which are very similar to that obtained in Israeli children, suggesting a consistency of SOS across ethnicities.

Keywords Bone mineral content $\cdot$ Ethnicity . Quantitative ultrasound · Reference data $\cdot$ Speed of sound

\section{Introduction}

Osteoporosis is defined as a systemic skeletal disease characterized by low bone mass and microarchitectural deterioration of bone tissue with a consequent increase in bone fragility [1]. More than $85 \%$ of peak skeletal mass is accrued by the age of 18 years. Failure to achieve peak bone mass during this critical period can not be compensated for later in life [2]. Therefore, it is important to have reliable tools and methods to assess bone health during childhood and adolescence.

Measurement of bone mineral content (BMC) by dualenergy $\mathrm{x}$-ray absorptiometry (DEXA) is the most commonly used method for the assessment of bone health in children and adults. However, areal DEXA measurements are influenced by the changes in bone size that occur with growth in children making the interpretation of change in true BMC difficult [3, 4].

Recently, new technologies have added to the repertoire of available tools for bone-health assessment in children [3-5]. Quantitative ultrasonography (QUS) in the diagnosis and evaluation of osteoporosis-associated fractures are gaining popularity $[4,5]$. QUS technique has the 
advantages of being simple, relatively inexpensive, portable, non-invasive and ionizing radiation-free. It has been claimed that measurement of speed of sound (SOS) along the length of a long bone also gives information about not only the bone density, but also the micro-architecture, cortical thickness and elasticity of the bone [5-8]. However, experience, normative data and comparison with the established methods are lacking regarding the use of QUS in children. In addition to that, there is a need for locally established reference values due to ethnical, geographic and nutritional differences in various populations. Our aim was to establish the reference database for SOS at the radius and tibia in healthy Turkish children and investigate the relationship between SOS Z-score and height, weight, daily calcium (Ca) intake, alkaline phosphatase (ALP) and osteocalcin in a randomly selected subgroup of children.

\section{Method}

The study was approved by the Ethics Committee of the Marmara University Medical School. Informed consent was obtained from the parents of each child or from the child, if the child was older than 16 years of age. In the first part of the study, 0-16-year-old healthy subjects were recruited from the well child clinics of Marmara University Hospital, and neighboring primary and high schools. Regarding the schoolchildren, after permission for the study was obtained from school administrators, detailed questionnaires and consent forms for the study were distributed to randomly selected classrooms 1 week prior to the study. The questionnaire included the medical and nutritional history of each child. At the day of the study, the research team visited the schools, collected the questionnaires and consent forms that had been filled in and signed by the parents and performed the anthropometric and SOS measurements on those who had been given consent. Children were excluded if they had history of prematurity, intrauterine growth retardation, chronic systemic disease, chronic medication or height and weight standard deviation scores (SDSs) below $-2.0 \mathrm{SD}$.

The study population consisted of 463 healthy children. All subjects had their standing height measured by the same investigator using the same portable wall stadiometer (SECA) to the nearest $0.1 \mathrm{~cm}$. Weight was measured on a digital scale with a precision of $0.1 \mathrm{~kg}$ (SECA, model 708, Hamburg, Germany). Body mass index (BMI) was calculated as weight (kilograms) divided by height (meters) squared. Weight and height SDSs were calculated by using healthy Turkish children standards: patient's value minus mean of patient's age group divided by standard deviation of patient's age group [9].

SOS was measured in the distal third of the radius and the mid-shaft tibia using the Sunlight Omnisense 7,000 bone sonometer. Age- and gender-specific mean \pm SD SOS values for the tibia and radius were determined and compared with the sonometers' built-in original values obtained from the Israeli children.

Information on diet and $\mathrm{Ca}$ intake was calculated from self-reported 3-day nutritional diaries. Serum samples were collected for ALP and osteocalcin measurements from randomly selected subgroups of children $(n=124)$. Blood samples were drawn from an antecubital vein between $0800-1300 \mathrm{~h}$ and centrifuged after coagulation. Serum was stored at $-20^{\circ} \mathrm{C}$ until analysis. ALP was measured by a Roche/Hitachi analyzer, and osteocalcin was measured by immunometric assay (Immulite).

Table 1 The mean and standard deviation speed of sound (SOS) of tibia and radius by age and gender

\begin{tabular}{|c|c|c|c|c|c|c|}
\hline \multirow[t]{2}{*}{ Age (year) } & \multicolumn{3}{|c|}{ Girls } & \multicolumn{3}{|c|}{ Boys } \\
\hline & No. & Tibia & Radius & No. & Tibia & Radius \\
\hline $1-2$ & 26 & $3299.6 \pm 143.8$ & $3464 \pm 89.2$ & 28 & $3321.9 \pm 108.8$ & $3459.0 \pm 75.3$ \\
\hline 3 & 10 & $3519.1 \pm 76.6$ & $3579.7 \pm 90.3$ & 20 & $3532.4 \pm 108.2$ & $3636.9 \pm 91.6$ \\
\hline 4 & 16 & $3599.9 \pm 84.6$ & $3661.3 \pm 52.9$ & 21 & $3633.6 \pm 68.5$ & $3654.1 \pm 102.5$ \\
\hline 5 & 13 & $3605.6 \pm 69.1$ & $3616.8 \pm 96.8$ & 18 & $3648.6 \pm 91.7$ & $3668.0 \pm 88.8$ \\
\hline 6 & 23 & $3618.1 \pm 79.7$ & $3705.6 \pm 95.3$ & 13 & $3661.5 \pm 93.1$ & $3739.2 \pm 81.9$ \\
\hline 7 & 24 & $3648.1 \pm 87.8$ & $3746.2 \pm 94.4$ & 21 & $3665.9 \pm 88.5$ & $3711.9 \pm 99.6$ \\
\hline 8 & 23 & $3641.4 \pm 75.1$ & $3712.0 \pm 68.6$ & 18 & $3673.5 \pm 87.5$ & $3701.1 \pm 75.7$ \\
\hline 9 & 30 & $3641.6 \pm 91.9$ & $3725.4 \pm 75.3$ & 10 & $3643.3 \pm 82.7$ & $3705.6 \pm 86.8$ \\
\hline 10 & 16 & $3650.5 \pm 91.4$ & $3726.1 \pm 73.9$ & 26 & $3673.8 \pm 90.2$ & $3757.1 \pm 103.3$ \\
\hline 11 & 17 & $3618.9 \pm 76.2$ & $3711.1 \pm 119.2$ & 21 & $3672.5 \pm 102.5$ & $3715.4 \pm 95.4$ \\
\hline 12 & 10 & $3716.4 \pm 120.9$ & $3725.9 \pm 80.7$ & 11 & $3658.7 \pm 114.1$ & $3708.6 \pm 130.4$ \\
\hline 13 & 15 & $3797.0 \pm 135.3$ & $3794.1 \pm 119.2$ & 10 & $3742.0 \pm 111.6$ & $3745.3 \pm 87.2$ \\
\hline $14-16$ & 11 & $3823.4 \pm 94.4$ & $3862.2 \pm 131.2$ & 12 & $3669.6 \pm 77.5$ & $3730.5 \pm 63.3$ \\
\hline
\end{tabular}




\section{Statistics}

For reference data, after obtaining descriptive statistics (mean, SD) for each age group, scatter diagrams of the data were constructed. Age was taken as an independent variable, while SOS measurements were taken as dependent variables. The distribution of the data was analyzed by the curve-fitting method, the least squared residuals that best fit the polynomial per age group. The different slopes observed in SOS results by age within the study groups enforced fitting a series of different polynomials. The fit was carried out by finding the set of parameters which minimized the sum of the squared residuals.

Pearson correlations were used to analyze the relationship between the variables. Age-dependent variables were converted to Z-scores before being used in the correlation analyses to eliminate the effect of interdependency among the variables. $Z$-scores were calculated using the formula: $\mathrm{Z}$-score $=(\mathrm{X}$-average $\mathrm{X}) / \mathrm{SD}$.

\section{Results}

Four hundred and sixty-three $(n=463)$ children (234 females, 229 males) were enrolled in the study. The mean age of the study population was $7.72 \pm 0.08$ years. The mean \pm SD of SOS values of the tibia and radius in girls and boys for different ages are seen in Table 1 .

A steep increase in mean tibia SOS values was seen at 1-4 years in girls with a total increment of $300 \mathrm{~m} / \mathrm{s}$ during this period. Similarly, radius SOS values also showed a steep increase at ages 1 thorough 6 with a total increment of $241 \mathrm{~m} / \mathrm{s}$ during this time period. From 6 years through 10 years, SOS values showed a very slow increase followed by a second period of steep increase after age 11 for both the tibia and radius (Figs. 1 and 2).

A similar pattern was observed in boys, demonstrating a steep increase of $340 \mathrm{~m} / \mathrm{s}$ between 1-4 years of age in reference to the tibia, very slow increase between ages 7-12 and a second period of acceleration in SOS measurements after 12 years of age. Individual SOS values and fitted points for the mean SOS are seen in Figs. 1 and 2 in
Fig. 1 Individual plots and fitted means of tibia SOS measurements in Turkish children $(T R)$ in comparison with fitted means of original data retrieved from Israeli $(I L)$ children in girls (a) and boys (b). The fitted points for the mean SOS was the results of the curve fitting and slightly differs from the mean SOS values listed in Table 1 a

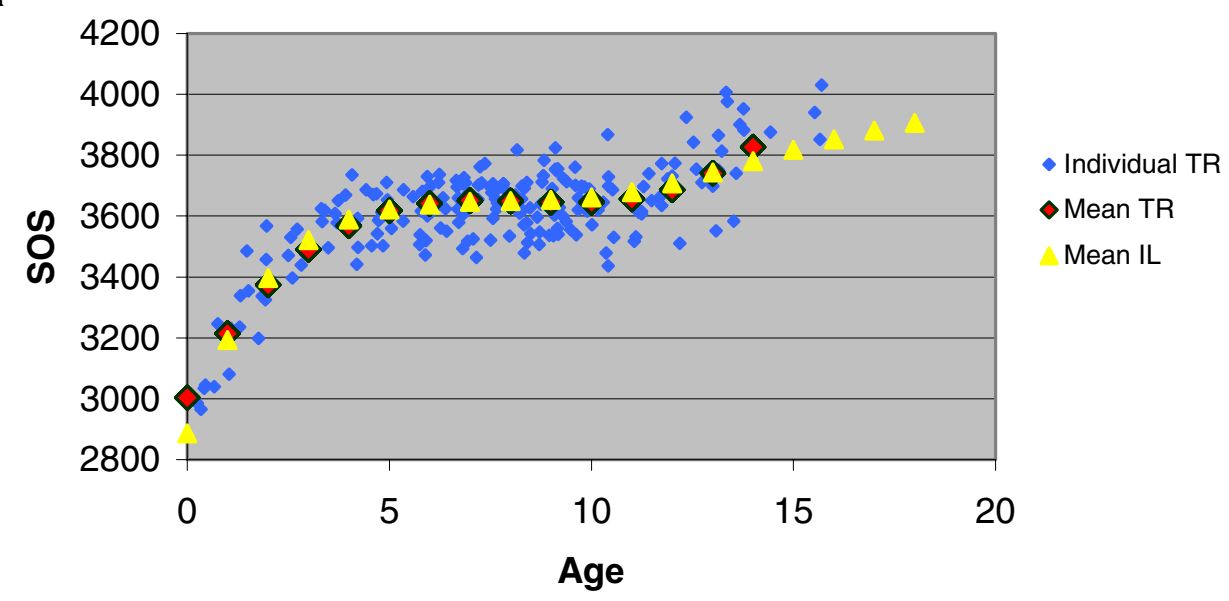

b

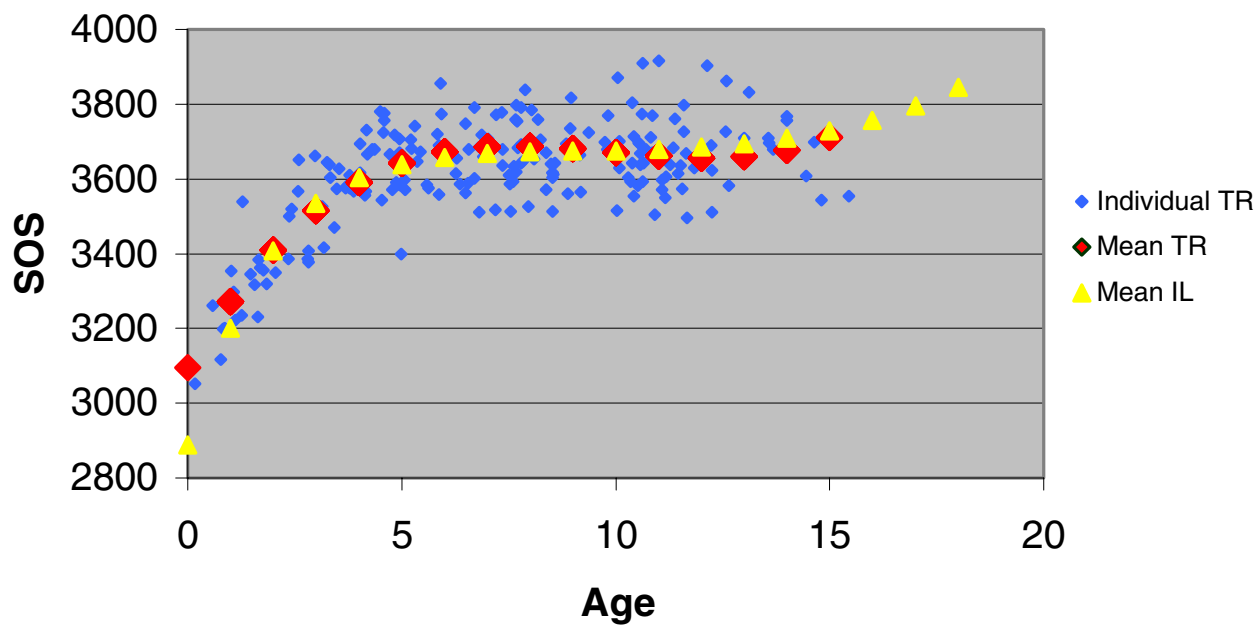


comparison with values obtained from Israeli children. Tibial SOS measurements were very similar in both populations. However, regarding the radius, our population has slightly lower mean SOS values in comparison with Israeli data. The average differences in Z-scores were -0.1 SDS in ages 3-7 years and -0.7 SDS in ages $8-15$ years in girls and -0.2 SDS in ages $3-7$ years and -0.8 SDS in ages $8-15$ in boys.

\section{Correlations between QUS measurements and other parameters}

A significant correlation between tibia $\mathrm{Z}$-score and radius Z-score in both genders was noted (girls $r=0.40, p<0.0001$; boys $r=0.33, p<0.0001$ ). In girls, but not in boys, weight SDS correlated negatively with tibia Z-scores $(r=-0.24$, $p<0.001)$. Height SDS, osteocalcin levels and daily $\mathrm{Ca}$ intake did not correlate with tibia and radius Z-scores in both genders. Analysis of the combined group (girls and boys) showed a significant negative correlation between ALP and both radius and tibia Z-scores (tibia: $p=0.01$, radius: $p<0.05)$. However, when the data was analysed separately, the correlation was significant only in boys $(r=-0.35, p<0.01)$.

\section{Discussion}

We have established pediatric reference data for SOS regarding the radius and tibia measured by quantitative ultrasonography in our population. The results revealed a triphasic increase in the SOS values in both the tibia and radius in both sexes from ages 1 through 16 years. There is a steep increase in SOS during the first 4-7 years of life followed by a very slow increase until age 11 in girls and 12 in boys and another steep increase thereafter. Although we did not evaluate the pubertal status of the study subjects, the timing of the second increase in SOS coincides with the onset of puberty in our population. This pattern in bone measurement is physiologically sound as it resembles the pattern of growth rate and bone acceleration [10]. There are two periods during which growth is accelerated: during the first two years of life and during adolescence [2].
Fig. 2 Individual plots and fitted means of radius SOS measurements in Turkish children $(T R)$ in comparison with fitted means of original data retrieved from Israeli $(I L)$ children in girls (a) and boys (b). The fitted points for the mean SOS was the results of the curve fitting and slightly differs from the mean SOS values listed in Table 1 a

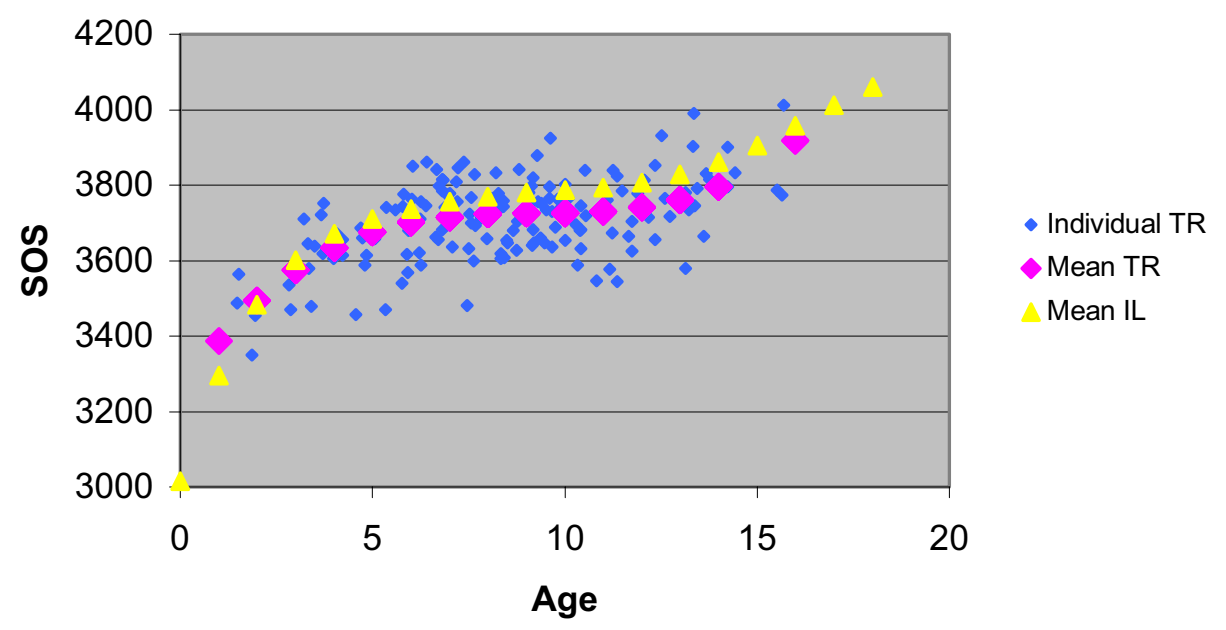

b

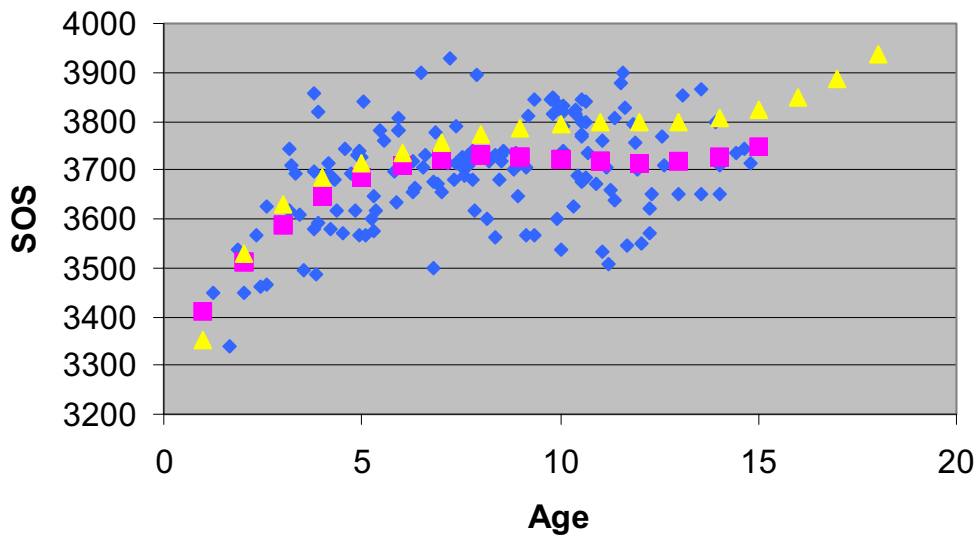

- Individual TR Mean TR Mean IL 
Various studies have been done to establish a reference database for SOS at multiple sites in different ethnic groups using the Sunlight Omnisense [6, 8]. However, the present study is the second reference study regarding the use of this technique in children. Zadik et al. [8], in their first reference study, also demonstrated a triphasic pattern in SOS values in Israeli children. Minor differences were observed between the two cohorts such as slightly lower radial SOS values after 8 years in the Turkish cohort. Apart from this difference, mean SOS values were almost identical in two cohorts. Similar patterns and SOS values were obtained in two populations, suggesting consistency of SOS across ethnicities.

We have also evaluated the relationship between SOS Z-scores and height SDS, weight SDS, daily $\mathrm{Ca}$ intake, osteocalcin and ALP levels in a randomly selected subset of the patients. There were no correlations between height SDS and either tibia or radius SOS Z-scores. However, some studies have claimed that height and weight were significantly correlated with bone SOS measurements, since all three parameters are highly covariate and dependent on age, one must use age-adjusted SDS scores for this purpose [5]. Height and weight SDSs were used for correlation analyses with SOS Z-scores in our study. We detected a negative correlation between weight SDS in girls and tibia Z-scores, whereas no such correlation was observed between weight SDS and radius Z-scores. This could be due to increased subcutaneous fat in overweight girls hampering the measurement of SOS and causing low SOS for the tibia. Supporting our observation, Eliakim et al. [11] demonstrated a reduction in bone strength measured by QUS in obese children.

We did not find any correlation between daily Ca intake and SOS Z-scores. Zadik et al. [8] also did not find such a relationship in Israeli children. Average daily Ca intake was similar in both cohorts $(530 \pm 262 \mathrm{mg} /$ day in Israel and $578 \pm 293 \mathrm{mg} /$ day in the present study). The study done by Kardinal et al. [12] in six European countries to investigate the association between $\mathrm{Ca}$ intake and radial bone density by DEXA also found no correlation between $\mathrm{Ca}$ intake and BMD, except for one country where BMD became inversely associated with $\mathrm{Ca}$ intake.

There was no significant correlation between osteocalcin and SOS Z-scores, but a negative correlation was demonstrated with ALP and tibia SOS Z-scores in the combined group. Analysis of the boys and girls separately showed that this correlation existed only in boys. Although we did not measure vitamin D levels, it is tempting to speculate that the inverse relationship between ALP and SOS values might suggest subclinical vitamin D deficiency and/or insufficient $\mathrm{Ca}$ intake in some subjects. Calculated daily $\mathrm{Ca}$ intakes in this study are suboptimal for pubertal age groups.
In conclusion, the present study establishes a pediatric reference data for tibia and radius SOS in our population which is very similar to built-in data obtained in the Israeli population, suggesting SOS measurements do not vary considerably between the populations. The QUS technique has the advantages of being simple, relatively inexpensive, portable, non-invasive and ionizing radiation-free. It has been claimed that measurement of speed of sound (SOS) along the length of a long bone also gives information about not only the bone density, also the micro-architecture, cortical thickness and elasticity of the bone [5-8]. However, evaluation of QUS in bone diseases still need to be investigated.

Acknowledgements This work was supported by the Turkish Academy of Sciences, in the framework of the Young Scientist Award Program (EA-TUBA-GEBIP/ 2001-1-1).

We thank Irit Yaniv and Tseela Schwartz for assistance in statistics and comparison with Israeli data.

\section{References}

1. Steelman J, Zeitler P (2001) Osteoporosis in pediatrics. Pediatr Rev 22:56-65

2. Bailey DA, McKay HA, Mirwald RL, Crocker PR, Faulkner RA (1999) A six-year longitudunal study of the relationship of physical activity to bone mineral accrual in growing children: The University of Saskatchewan Bone Mineral Accrual Study. J Bone Miner Res 14:1672-1679

3. Leonard MB, Zemel BS (2002) Current concepts in pediatric bone disease. Pediatr Clin North Am 49:143-173

4. Gilsanz V (1998) Bone density in children: a review of the available techniques and indications. Eur J Radiol 26:177-182

5. Weiss M, Ben-shlomo A, Hagag P, Rapoport M (2000) Reference database for bone speed of sound measurement by a novel quantitative multi-site ultrasound device. Osteoporos Int 11:688 696

6. Hayman SR, Drake WM, Kendler DL, Olszynski WP, Webber CE, Rosen CJ, Genant HK, Orwoll ES, Pickard LE, Adachi JD (2002) North American male reference population for speed of sound in bone at multiple skeletal sites. J Clin Densitom 5:63-71

7. Njeh CF, Hans D, Wu C, Kantorovich E, Sister M, Fuerst T, Genant HK (1999) An in vitro investigation of the dependence on sample thickness of the speed of sound along the specimen. Med Eng Phys 21:651-659

8. Zadik Z, Price D, Diamond G (2003) Pediatric reference curves for multi-site quantitative ultrasound and its modulators. Osteoporos Int 14:857-862

9. Neyzi O, Yalcindag A, Alp H (1973) Heights and weights of Turkish children. J Trop Pediatr Environ Child Health 19:5-13

10. Bachrach LK (2001) Acquisition of optimal bone mass in childhood and adolescence. Trends Endocrinol Metab 12:22-28

11. Eliakin A, Nemet D, Wolach B (2001) Quantitative ultrasound measurements of bone strength in obese children and adolescents. J Pediatr Endocrinol Metab 14:159-164

12. Kardinaal AF, Ando S, Charles P, Charzewska J, Rotily M, Vaananen K, Van Erp-Baart AM, Heikkinen J, Thomsen J, Maggiolini M, Deloraine A, Chabros E, Juvin R, Schaafsma G (1999) Dietary calcium and bone density in adolescent girls and young women in Europe. J Bone Miner Res 14:583-592 